\title{
DE-1 versus Cluster spectral density observations: the effect on ion outflow above the polar cap
}

\author{
Hamza A. Abudayyeh ${ }^{\star}$ Imad A. Barghouthi, Ghadeer Al-Sarsour, Husain Alsamamra
}

Al-Quds University, Department of Physics, Jerusalem, Palestine

\author{
Article history \\ Received May 25, 2015; accepted July 23, 2015. \\ Subject classification: \\ Wave-particle interaction, Ion outflow, Polar wind, Central polar cap, Cusp, Polar cap, Diffusion coefficients, Cluster, DE-1.
}

\section{ABSTRACT}

Wave-particle interaction is a very important mechanism in describing the outflow of ions at high latitudes and high altitudes. Quasi-linear perpendicular velocity diffusion coefficients are used to describe the effect of wave-particle interactions, therefore it is essential to determine the correct diffusion coefficients that must be used to model the outflow of ions. In this study a Monte Carlo method is used to assess the role of different diffusion coefficients for $\mathrm{O}^{+}$and $\mathrm{H}^{+}$ions at high altitudes above the polar cap. Two different sets of diffusion coefficients obtained from Barghouthi [1997]; Barghouthi et al. [1998] and Nilsson et al. [2013] are used. Barghouthi [1997]; Barghouthi et al. [1998] used spectral density measurements from Dynamic Explorer 1 spacecraft (DE-1) observations to calculate the diffusion coefficients, while Nilsson et al. [2013] used spectral density measurements from the Cluster spacecraft to obtain the diffusion coeffcients. It was found that diffusion coefficients from Barghouthi [1997]; Barghouthi et al. [1998] in the cusp (aurora) and central polar cap (polar wind) respectively, describe well the observations of ion outflow at altitudes lower than $5 R_{E}$, but yield unreasonably high parallel velocities and temperatures at higher altitudes. Also diffusion coefficients from Cluster spectral density measurements produce reasonable results for high altitudes and unreasonably low parallel velocities and temperatures for the low altitude region. Therefore it is suggested that a combination of these diffusion coefficients is used where the diffusion coefficients given by Barghouthi [1997]; Barghouthi et al. [1998] are used at low altitudes and the diffusion coefficients obtained from Cluster measurements are used at high altitudes.

\section{Introduction}

The outflow of plasma from ionospheric origin along open geomagnetic field lines above the polar cap is subject to intense investigation. It is well observed that ions have high perpendicular temperatures at high altitudes despite the presence of perpendicular adiabatic cooling [Huddleston et al. 2000, Bouhram et al. 2004, Nilsson et al. 2004, Nilsson et al. 2006]. This is an indication of a perpendicular heating mechanism that acts at high altitudes. Arvelius et al. [2005] showed using the
Cluster CIS / CODIF instrument that at 8-12 $\mathrm{R}_{E}$ geocentric distance the distribution of $\mathrm{O}^{+}$outflows cannot be explained by velocity filter dispersion only. This is a clear suggestion of further additional energization processes for outflowing $\mathrm{O}^{+}$ions at high altitudes and high latitudes in the dayside polar region. Observations indicated a significant level of electromagnetic turbulence at high altitudes above the polar cap [Gurnett et al. 1984, Gurnett and Inan 1988, Nilsson et al. 2012, Waara et al. 2012] which suggested an interaction known as wave-particle interaction (WPI)[Chang and Coppi 1981, Chang et al. 1986, Retterer et al. 1987, Crew et al. 1990, Retterer et al. 1994, Barghouthi 1997, Barghouthi et al. 1998, Bouhram et al. 2004, Waara et al. 2010, Slapak et al. 2011, Waara et al. 2011, Waara et al. 2012]. Field aligned acceleration mechanisms such as field aligned electric fields [Lundin et al. 1995, Maggiolo et al. 2006] and centrifugal acceleration [Cladis 1986, Horwitz et al. 1994, Demars et al. 1996, Nilsson et al. 2008, Nilsson et al. 2010] were also investigated to explain the energization up to kiloelectronvolt energies of $\mathrm{O}^{+}$ions above the polar cap.

The properties of an ion species may be determined from its distribution function $f_{s}(\mathbf{r}, \mathbf{v}, t)$ where the development of the distribution function is governed by the Boltzmann equation [Schunk 1977]:

$$
\frac{\partial f_{s}}{\partial t}+\mathbf{v}_{\mathbf{s}} \cdot \nabla f_{s}+\frac{1}{m_{s}} \mathbf{F} \cdot \nabla_{\boldsymbol{v}_{s}} f_{s}=\frac{\delta f_{s}}{\delta t}
$$

where $\mathbf{v}_{\mathbf{s}}, m_{s}$ are the velocity and mass of the $s^{\text {th }}$ species respectively; $\nabla, \nabla_{v_{s}}$ are the gradient in space and velocity coordinates respectively; $\mathbf{F}$ is the total external force acting on the ion species (for example, the ambipolar electric force, mirror force, and gravity); and $\frac{\delta f_{s}}{\delta t}$ represents the rate of change of the distribution function in a region of phase space due to collisions and wave-particle interactions. 
At altitudes considered in this study the plasma is tenuous enough to neglect collisions. In such case the right hand side of Equation (1) reduces to [Retterer et al. 1987]:

$$
\frac{\delta f_{s}}{\delta t}=\frac{1}{v_{\perp s}} \frac{\partial}{\partial v_{\perp s}}\left(D_{\perp s} v_{\perp s} \frac{\partial f_{s}}{\partial v_{\perp s}}\right)
$$

where $v_{\perp s}$ is the component of the velocity of the $\mathrm{s}^{\text {th }}$ species that is perpendicular to the geomagnetic field and $D_{\perp s}$ is the quasi-linear velocity diffusion coefficient for the $\mathrm{s}^{\text {th }}$ species. The perpendicular velocity diffusion coefficient may be written as [Retterer et al. 1987]:

$$
D_{\perp s}=\left(\frac{q_{s}}{2 m_{s}}\right)^{2} S_{L}
$$

where $q_{s}$ is the charge of the $s^{\text {th }}$ ion species and $S_{L}$ is the electric field spectral density at the ion gyrofrequency that is efficient in heating the ions. $S_{L}$ is related to the total spectral density at the ion gyrofrequency $(S)$ by $S_{L}=\eta S$, where $\eta$ is the fraction of spectral density at the gyrofrequency of the ion that is efficient in heating the plasma. At lower altitudes (few $\mathrm{R}_{E}$ ), values for $\eta$ ranging from a few percent to $10 \%$ were used in several studies [Bouhram et al. 2004, Barghouthi and Atout 2006]. Nilsson et al. [2013] found that for geocentric distances between 8 and $10 \mathrm{R}_{E}$ in the central polar cap (CPC) and cusp, good agreement was attained for a value of $\eta=\frac{1}{2}$.

The relation between perpendicular temperature and wave activity is determined by two competing effects: the sporadic nature of the waves and how dominating the most recent heating is. A good correlation could be established if the recent heating is dominating, and higher sporadic wave activity can be tolerated. If ion heating is not sporadic then recent heating does not necessarily need to dominate in order to obtain a good correlation between ion temperature and wave intensity. Therefore sporadic heating can explain a region of high temperature and relatively low wave activity. Waara et al. [2012] studied this relation and found that there is indeed a correlation except at the highest perpendicular temperatures which indicates that the strongest heating is most likely sporadic.

Barghouthi et al. [1998] used data from the Plasma Wave Instrument on board Dynamics Explorer 1 (DE-1) to estimate the perpendicular diffusion coefficients in the polar wind for both $\mathrm{O}^{+}$and $\mathrm{H}^{+}$ ions. Four sets of data were chosen that cover altitude ranges from 1.5 to $4.5 \mathrm{R}_{E}$ at polar latitudes and sample both polar caps. Using these spectral densities, and assuming a value of $\eta=\frac{1}{8}$, the perpendicular diffusion coefficients were calculated using Equation (3) and were found to be:

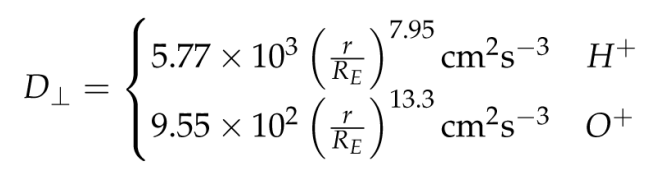

In the auroral region Barghouthi [1997] assumed that the altitude dependence of the spectral density to resemble that in the polar wind [Barghouthi et al. 1998]. By using the altitude dependence from Barghouthi et al. [1998] and the measured spectral density at auroral latitudes at 2.0 $\mathrm{R}_{E}$ from Retterer et al. [1987], the diffusion coefficients for $\mathrm{O}^{+}$and $\mathrm{H}^{+}$ion species were calculated. The diffusion coefficient for the ions in the auroral region may be given by:

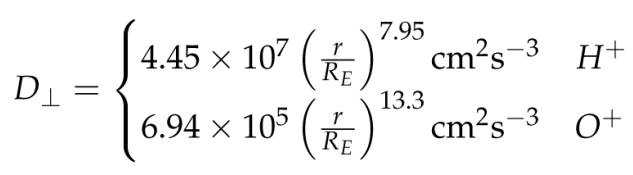

Waara et al. [2011] used data from three instruments on board the Cluster spacecraft namely: EFW (Electric Field and Wave experiment), FGM (Fluxgate Magnetometer) and CIS (Cluster Ion Spectrometry experiment). The data set consisted of EFW and FGM wave data above $5 \mathrm{R}_{E}$ when outflowing $O^{+}$ions were seen in the energy spectrograms of CIS. Using this data the average spectral density above the polar cap was calculated and estimates for the diffusion coefficients were obtained. Nilsson et al. [2012] further subdivided this data set into three regions with respect to their latitude: the central polar cap (CPC), nightside polar cap, and cusp. Using these values for the electric field wave activity near the $\mathrm{O}^{+}$gyrofrequency, Nilsson et al. [2013] used Equation (3) to calculate the diffusion coefficients in each region.

In this study we will use spectral density measurements at the $\mathrm{H}^{+}$gyrofrequency from Nilsson et al. [2012] to calculate the diffusion coefficients for $\mathrm{H}^{+}$ions based on Cluster observations. Then we will use a onedimensional kinetic model that includes the effects of ambipolar electric field, wave-particle interaction, gravitational and mirror force to investigate the effect of varying the diffusion coefficients from those based on DE-1 spectral density measurements to those based on Cluster measurments for altitudes up to $15 \mathrm{R}_{E}$. Two flux tubes called central polar cap (CPC) and cusp were taken to be along geomagnetic field lines calculated by using Tsyganenko T96 model Tsyganenko [1995, 1996]. In such case we will be assuming that the diffusion coefficients from DE-1 measurements are valid for all altitudes including those higher than $5 \mathrm{R}_{E}$ and the diffusion coefficients from Cluster measurements are valid for all altitudes including those less than $5 \mathrm{R}_{E}$ which is just an extrapolation for each data set. In performing such a 
calculation we aim to provide modelers using more sophisticated 3 dimensional models with proper diffusion coefficients for their studies.

This paper will be organized as follows: Section 2 includes a brief description of the model used. In Section 3 we will present our results. Discussions and conclusions will be presented in Section 4 .

\section{Method}

This study is an extension to Barghouthi [2008] in the aurora and Barghouthi et al. [2011] in the polar cap. A Monte Carlo method was used to study the outflow of $\mathrm{O}^{+}$and $\mathrm{H}^{+}$ions for geocentric distances from 1.7 to 14.7 $\mathrm{R}_{E}$ in the CPC and from 1.2 to $15.2 \mathrm{R}_{E}$ in the cusp. The flight trajectories that we call CPC and cusp are defined in Nilsson et al. [2012]. Aside from the modification of the flight trajectories three other modifications on the Barghouthi model were conducted:

1 . The radial magnetic field was replaced by a magnetic field calculated by the T96 model [Tsyganenko 1995, 1996].

2. The perpendicular velocity diffusion coefficients were varied such that the set based on DE-1 spectral density measurements were first used and then the set based on Cluster spectral density measurement were used.

3. The boundary conditions were taken to be as follows:

- The $\mathrm{O}^{+}$velocities at the exobase were taken to be 2.0 and $0.5 \mathrm{~km} / \mathrm{s}$ for the CPC and cusp respectively [Nilsson et al. 2013]. For $\mathrm{H}^{+}$ions the initial velocities were taken to be 18 [Nilsson et al. 2013] and $16 \mathrm{~km} / \mathrm{s}$ [Barghouthi 1997] for the CPC and cusp respectively.

- The densities at the lower altitude boundary were taken to be 10 and $10,000 \mathrm{~cm}^{-3}$ for $\mathrm{O}^{+}$ions for the CPC and cusp respectively [Nilsson et al. 2013] and 34 [Nilsson et al. 2013] and $200 \mathrm{~cm}^{-3}$ [Barghouthi 1997] for $\mathrm{H}^{+}$ ions in the CPC and cusp respectively.

- The electron temperature in addition to the temperature of both ions were assumed to be $3000 \mathrm{~K}$ at the exobase [Barghouthi et al. 1998].

The ions are injected at the lower altitude boundary $\left(r_{0}=1.7 R_{E}\right.$ for the CPC and $r_{0}=1.2 R_{E}$ for the cusp) with random velocities consistent with a drifting Maxwellian distribution. The ion motion is tracked for a small time interval $\Delta t$ as it moves under the effect of the ambipolar electric field and the gravitational and mirror forces. The influence of wave-particle interaction (WPI) was simulated by changing the perpendicular velocity of the ion by a random increment $\Delta v_{\perp}$ such that $\Delta v_{\perp}=4 D_{\perp} \Delta t$. The flux tube is divided into bins (130 bins for the CPC and 140 bins for the cusp) which act as recorders. When an ion enters one of these bins its parallel and perpendicular velocities are

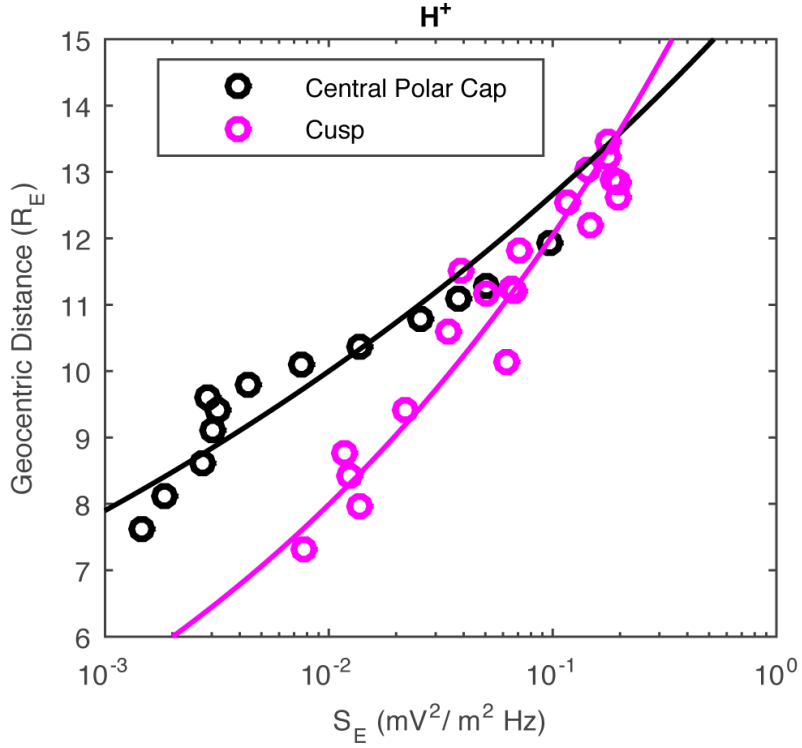

Figure 1. $\mathrm{H}^{+}$observed electric field spectral densities $\left[(\mathrm{mV} / \mathrm{m})^{2} / \mathrm{Hz}\right]$ at different geocentric distances $\left[\mathrm{R}_{E}\right]$ are shown by circles for the central polar cap (black) and cusp (magneta). Power law fits to the data are shown with solid lines.

registered for later use. This procedure is repeated for a large number of ions $\left(10^{5}-10^{7}\right.$ ions) until they exit either from the bottom or the top of the flux tube. Finally the registered data are used to calculate the moments and distribution functions of both ions. However, since the ambipolar electric field depends on the number densities of the ions which are outputs of the simulation, an iterative approach is used until convergence is achieved. For a more detailed description on how the moments and distribution functions are calculated see Barghouthi et al. [2003].

\section{Results}

The spectral density at the $\mathrm{H}^{+}$gyrofrequency was obtained from Nilsson et al. [2012] and is shown in Figure 1 for the central polar cap and cusp. Using a power law fit and Equation (3) the diffusion coefficients for $\mathrm{H}^{+}$ ions were found to be $D_{\perp}\left(H^{+}\right)=\eta 40\left(r / \mathrm{R}_{E}\right)^{9.77} \mathrm{~cm}^{2} \mathrm{~s}^{-3}$ for the central polar cap and $D_{\perp}\left(H^{+}\right)=\eta 2.02 \times 10^{6}\left(r / \mathrm{R}_{E}\right)^{5.61}$ $\mathrm{cm}^{2} \mathrm{~s}^{-3}$ for the cusp. Therefore, using the diffusion coefficients for $\mathrm{O}^{+}$ions obtained from Nilsson et al. [2013], the diffusion coefficients based on spectral densities measured on board Cluster spacecraft are given by

$$
D_{\perp}= \begin{cases}\eta 40\left(\frac{r}{R_{E}}\right)^{9.77} \mathrm{~cm}^{2} \mathrm{~s}^{-3} & H^{+} \\ \eta 1 \times 10^{5}\left(\frac{r}{R_{E}}\right)^{5.5} \mathrm{~cm}^{2} \mathrm{~s}^{-3} & O^{+}\end{cases}
$$

for the central polar cap and

$$
D_{\perp}= \begin{cases}\eta 2.02 \times 10^{6}\left(\frac{r}{R_{E}}\right)^{5.61} \mathrm{~cm}^{2} \mathrm{~s}^{-3} & H^{+} \\ \eta 5 \times 10^{4}\left(\frac{r}{R_{E}}\right)^{6.4} \mathrm{~cm}^{2} \mathrm{~s}^{-3} & O^{+}\end{cases}
$$



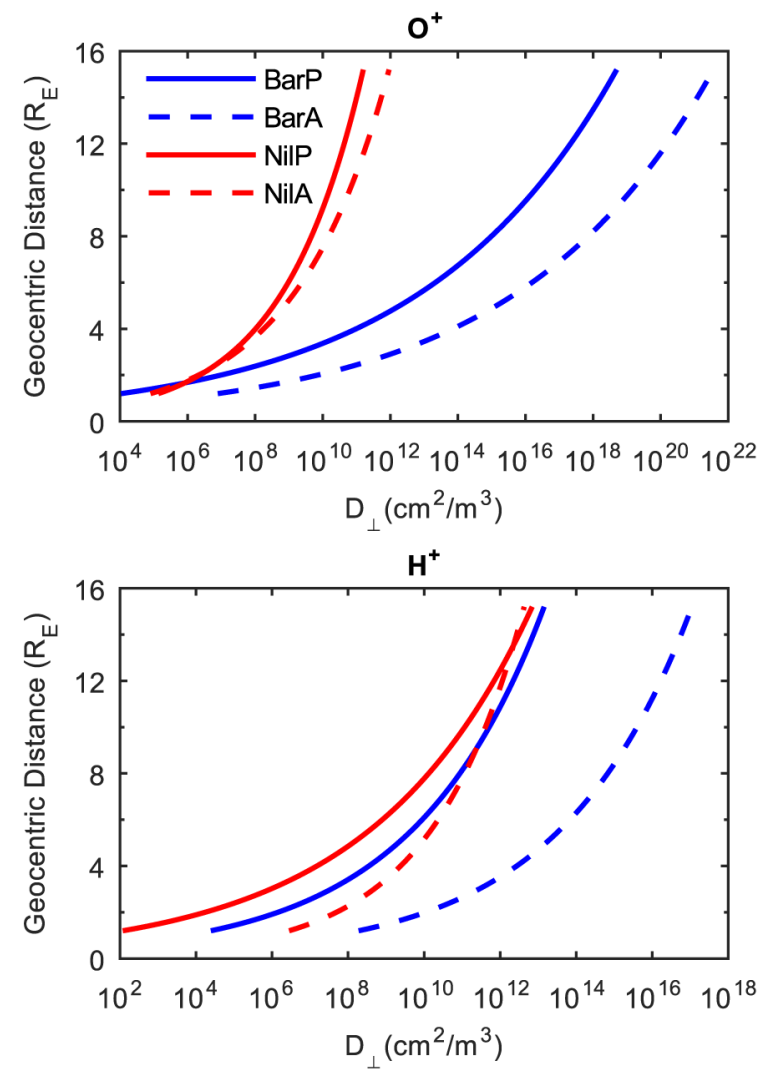

Figure 2. Perpendicular velocity diffusion coefficient profiles for $\mathrm{O}^{+}$ (top panel) and $\mathrm{H}^{+}$(bottom panel) ion species. BarP refers to diffusion coefficients calculated by Barghouthi et al. [1998] (Equation 4). BarA refers to the diffusion coefficients calculated by Barghouthi [1997] (Equation 5). NilP and NilA refer to diffusion coefficients in the central polar cap and cusp respectively calculated by Nilsson et al. [2013] (Equations 6 and 7 respectively).

for the cusp with a value of $\eta=\frac{1}{2}$ for both regions.

In this study we will present our simulation results for two sets of perpendicular velocity diffusion coefficients namely;

1. Diffusion coefficients by Barghouthi et al. [1998] in the polar wind and Barghouthi [1997] in the aurora given by Equations (4) and (5) respectively and the results due to these diffusion coefficients will hereinafter be denoted by BarP and BarA respectively.

2. Diffusion coefficients by Nilsson et al. [2013] in the CPC and cusp given by Equations (6) and (7) respectively and the results due to these diffusion coefficients will hereinafter be denoted by NilP and NilA respectively.

Figure 2 represents the perpendicular velocity diffusion coefficients for $\mathrm{O}^{+}$(top panel) and $\mathrm{H}^{+}$(bottom panel) ion species. The difference between the two sets is more significant for $\mathrm{O}^{+}$and at higher altitudes. For $\mathrm{O}^{+}$ ions the set calculated from DE-1 observations is nearly 5 orders of magnitude larger than those calculated by Cluster observations at $5 \mathrm{R}_{E}$ and nearly 8 orders of magnitude higher at $15 \mathrm{R}_{E}$. For $\mathrm{H}^{+}$ions the difference is more significant in the sunward part of the polar cap at higher altitudes as the diffusion coefficients given by Equation (5) (BarA) are higher than those given by Equation (7) (NilA) by 4 orders of magnitude at $15 \mathrm{R}_{E}$.

Figures 3 and 4 show the moments of $\mathrm{O}^{+}$and $\mathrm{H}^{+}$ ion species respectively. The density of $\mathrm{O}^{+}$ions at lower altitudes is nearly identical for both sets. At higher altitudes however, the density resulting from diffusion coefficients calculated from DE-1 spectral density measurements are smaller than those from Cluster observations. This is more evident in the BarP results since the initial density is three orders of magnitude less. One significant observation is that the profiles for NilP and NilA are identical even though the diffusion coefficients and initial densities are different. The profiles of $\mathrm{H}^{+}$density are only slightly affected by the change of diffusion coefficients.

The alternation of diffusion coefficients has a profound effect on the parallel bulk velocity of $\mathrm{O}^{+}$ions as it increases the bulk velocity by three orders of magnitude at the highest altitudes (from going from NilA to BarA for example). Therefore unrealistically high velocities are experienced when using diffusion coefficients calculated by DE-1 observations at high altitudes. On the other hand, at lower altitudes NilP and NilA diffusion coefficients produce relatively low bulk velocities at altitudes lower than $5 \mathrm{R}_{E}$, where the parallel bulk velocity of $\mathrm{O}^{+}$ions is only about $10 \mathrm{~km} / \mathrm{s}$. For the same set of diffusion coefficients, it may be noticed that the bulk velocity is higher in the more sunward part of the polar cap (BarA and NilA). $\mathrm{H}^{+}$bulk velocity is less affected by interchanging the diffusion coefficients. Nonetheless, at high altitudes high bulk velocities are experienced in BarP and BarA profiles.

The profiles for perpendicular and parallel temperatures also experience unusually high values at high altitudes for calculations based on DE-1 observations. For example, in the central polar cap region (polar wind) the perpendicular and parallel temperatures for $\mathrm{O}^{+}$ions are approximately $2 \times 10^{10}$ and $4 \times 10^{8} \mathrm{~K}$ at $10 \mathrm{R}_{E}$ for BarP profiles. While for NilP profiles at the same altitude the perpendicular and parallel temperatures are only about $2 \times 10^{6}$ and $9 \times 10^{4} \mathrm{~K}$ at the same altitude. The same holds for $\mathrm{H}^{+}$ions although with a much lesser extent. It may also be noted that the parallel bulk velocity, perpendicular and parallel temperature profiles for $\mathrm{H}^{+}$ions are decreasing or nearly constant up to altitudes of approximately $5 \mathrm{R}_{E}$ and increasing above that.

Figure 5 shows the contours for $\mathrm{O}^{+}$and $\mathrm{H}^{+}$distribution functions. As may be expected from WPI, conics in phase space are formed. In general it may be noticed that conics form at lower altitudes in BarA and BarP than in NilA and NilP. Also for the same set of diffusion coefficients conics form at more lower altitudes for $\mathrm{O}^{+}$ 
(a)

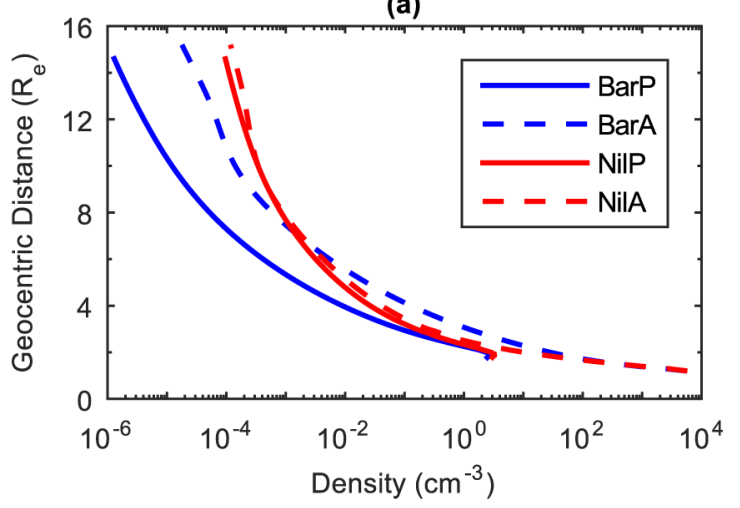

(c)

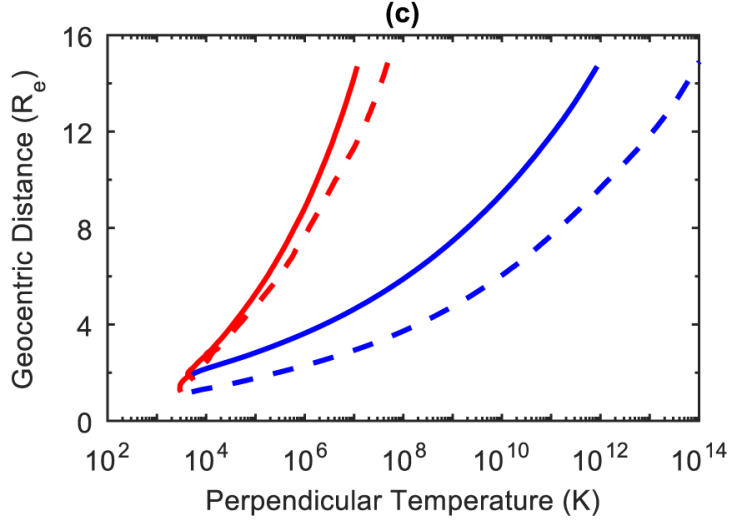

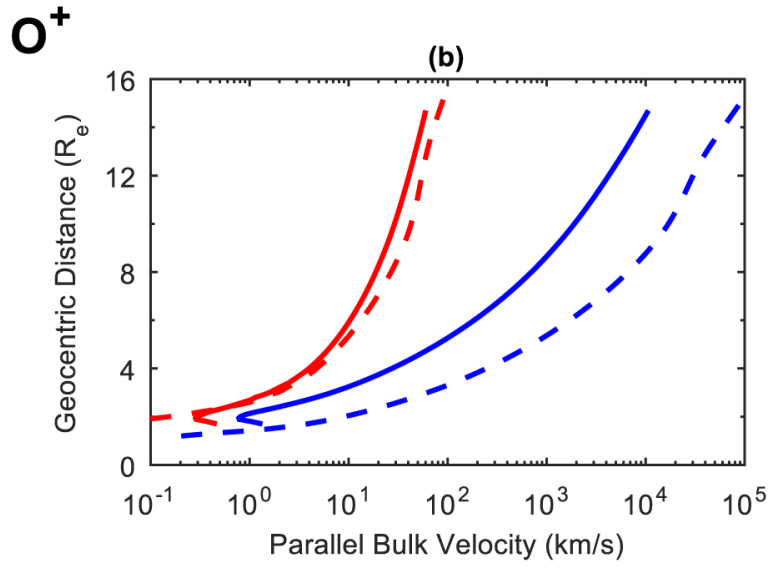

(d)

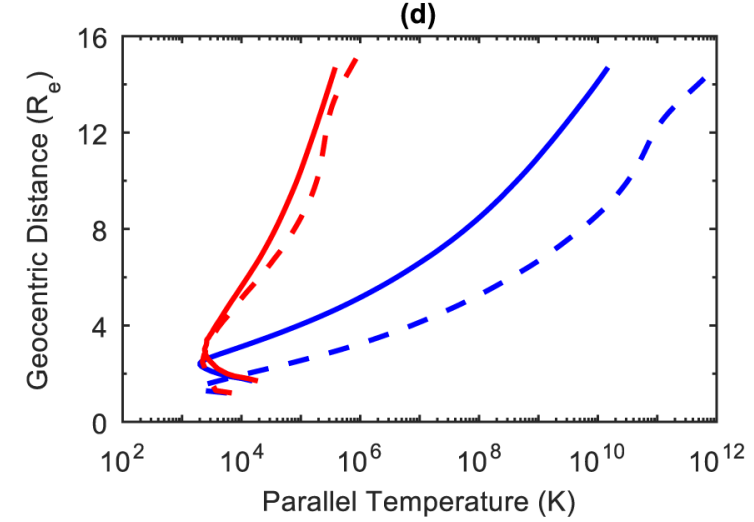

Figure 3. $\mathrm{O}^{+}$(a) density (b) parallel bulk velocity (c) perpendicular temperature and (d) parallel temperature profiles.

(a)

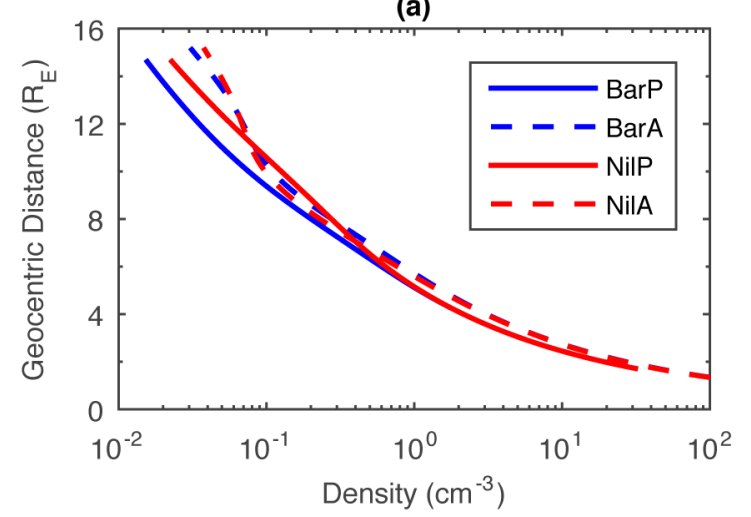

(c)

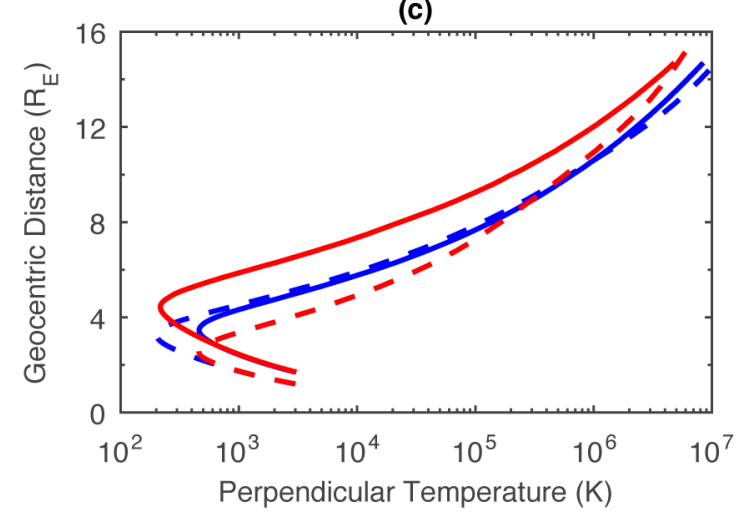

$\mathrm{H}^{+}$

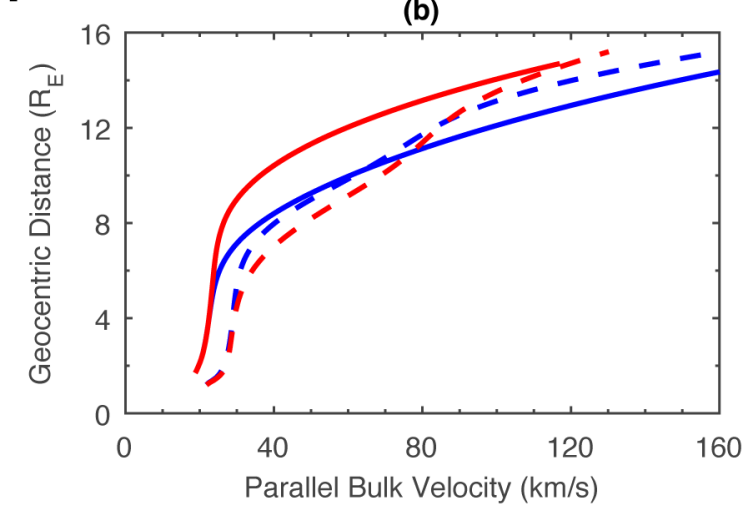

(d)

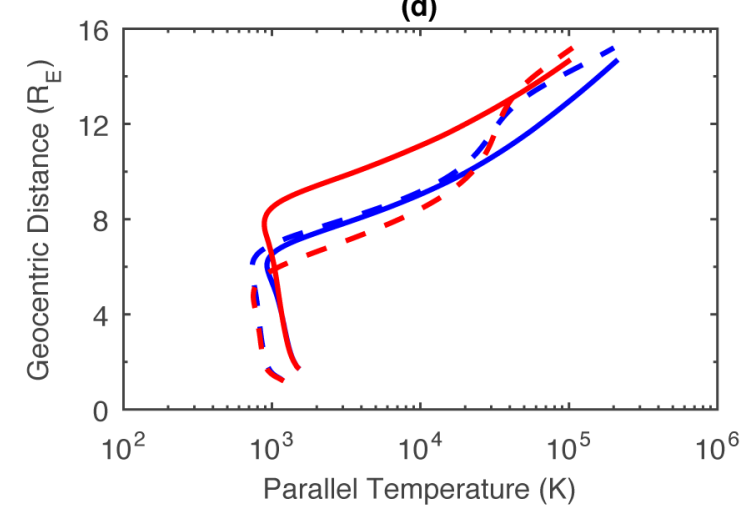

Figure 4. $\mathrm{H}^{+}$(a) density (b) parallel bulk velocity (c) perpendicular temperature and (d) parallel temperature profiles. 


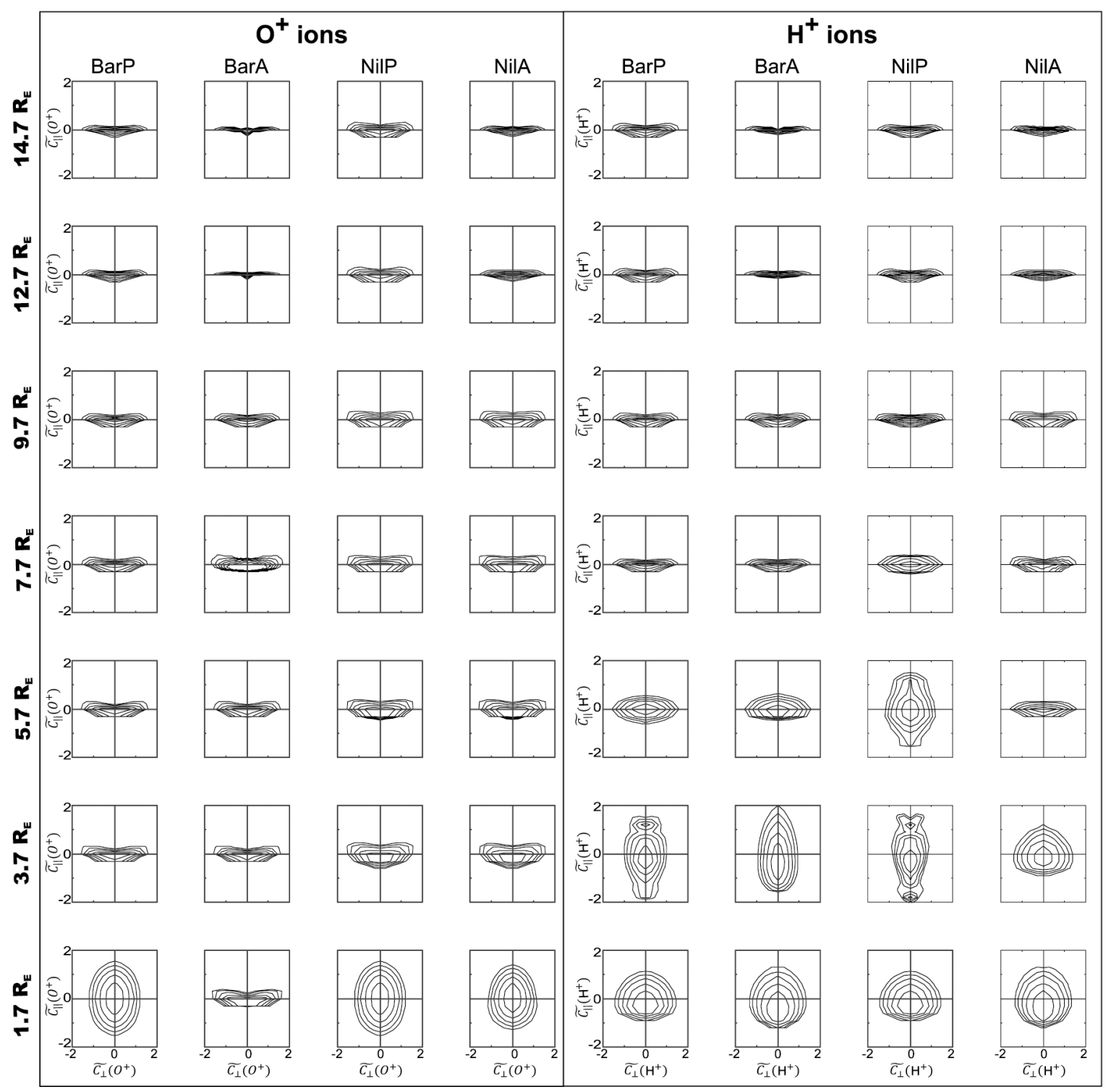

Figure 5. $\mathrm{O}^{+}$(first four columns) and $H^{+}$(columns 5-8) distribution function contours at 1.7, 3.7, 5.7, 7.7, 9.7, 12.7, and 14.7 $\mathrm{R}_{E}$, where $\mathrm{C}=$ $(\mathbf{v}-\mathbf{u}) / \sqrt{ } 2 \mathrm{kT} / \mathrm{m}$. The contours decrease successively by a factor of $e^{0.5}$ from the maximum.

ions in comparison with $\mathrm{H}^{+}$ions. Finally the latitude dependence is clear, since conics form at more lower altitudes in the most sunward regions of the polar cap.

\section{Discussion and conclusion}

As we noted in the previous section using diffusion coefficients calculated from DE-1 observations for high altitudes produces unreasonably high parallel velocities and parallel and perpendicular temperatures for $\mathrm{O}^{+}$ions species. The effect is less evident for $\mathrm{H}^{+}$ion outflows, but nonetheless it is notable for altitudes higher than $10 \mathrm{R}_{E}$. It is also evident that $\mathrm{O}^{+}$diffusion coefficients calculated based on Cluster spacecraft spectral density measurements are unsuitable for altitudes lower than $5 \mathrm{R}_{E}$.

To support our claim, Table 1 displays observational and simulation results for the CPC and cusp for $\mathrm{O}^{+}$ions. It is evident that using diffusion coefficients calculated from DE-1 spectral density observations yields results (BarP,BarA) that are near the observations at low altitudes, but that are not at all compatible with observations for higher altitudes.

The opposite may be said of results from using diffusion coefficients calculated based on spectral density measurements from Cluster data (NilP,NilA). These results agree with high altitude observations but are too low to explain low altitude behavior. This also suggests that the early history of ions along their trajectories is irrelevant for the strong heating region and only the most recent heating affects the moments of the ions. In other words most recent heating dominates. Therefore it may be anticipated that for the regions considered in this study, where the heating is mostly intense, ions with different initial conditions will have nearly the same velocities and temperatures at higher altitudes.

Due to the findings of this study we conclude that using one set of diffusion coefficients is not suitable to 


\begin{tabular}{|c|c|c|c|c|c|}
\hline Geocentric distance & Region & Characteristics & Observations & BarP/BarA & NilP/NilA \\
\hline $3 \mathrm{R}_{E}$ & Cusp & Temperature (eV) & $\begin{array}{l}\qquad 20-1000 \\
\text { Bouhram et al. [2004] }\end{array}$ & 740 (BarA) & 1 (NilA) \\
\hline $3 \mathrm{R}_{E}$ & Cusp & $\begin{array}{c}\text { Parallel bulk } \\
\text { velocity }(\mathrm{km} / \mathrm{s})\end{array}$ & $\begin{array}{l}\sim 25-100 \\
\text { Bouhram et al. [2004] }\end{array}$ & 63 (BarA) & 1.9 (NilA) \\
\hline $11.8 \mathrm{R}_{E}$ & Cusp & $\begin{array}{l}\text { Perpendicular } \\
\text { temperature }(\mathrm{eV})\end{array}$ & $\begin{array}{c}\sim 870-2280 \\
\text { Nilsson et al. [2013] }\end{array}$ & $8.24 \times 10^{8}(\mathrm{BarA})$ & 1109 (NilA) \\
\hline $11.8 \mathrm{R}_{E}$ & Cusp & $\begin{array}{c}\text { Parallel bulk } \\
\text { velocity }(\mathrm{km} / \mathrm{s})\end{array}$ & $\begin{array}{c}\sim 83 \\
\text { Nilsson et al. [2013] }\end{array}$ & $2.9 \times 10^{4}(\mathrm{BarA})$ & 54 (NilA) \\
\hline $2.3 \mathrm{R}_{E}$ & $\mathrm{CPC}$ & $\begin{array}{c}\text { Parallel bulk } \\
\text { velocity }(\mathrm{km} / \mathrm{s})\end{array}$ & $\begin{array}{l}\sim 1-2 \\
\text { Abe et al. [2004] }\end{array}$ & $1.5(\mathrm{BarP})$ & 0.5 (NilP) \\
\hline $10.1 \mathrm{R}_{E}$ & $\mathrm{CPC}$ & $\begin{array}{c}\text { Parallel bulk } \\
\text { velocity }(\mathrm{km} / \mathrm{s})\end{array}$ & $\begin{array}{c}\sim 56 \\
\text { Nilsson et al. [2013] }\end{array}$ & 2000 (BarP) & $30(\mathrm{NilP})$ \\
\hline
\end{tabular}

Table 1. Comparison between observational and simulation results for $\mathrm{O}^{+}$ions.

explain the outflow of $\mathrm{O}^{+}$and $\mathrm{H}^{+}$ions for all altitudes (at least those considered in this study). Hence some combination of these diffusion coefficients should be used where the diffusion coefficients given by Equations (4) and (5) should be used at low altitudes for the CPC and cusp respectively, and diffusion coefficients given by Equations (6) and (7) should be used for high altitudes in the CPC and cusp respectively. We cannot put a definite altitude at which the diffusion coefficients should be altered. It is important to note that we cannot suggest a given altitude to change the diffusion coefficient due to the following reasons:

1. Observations show that in the mid altitude region (5-7 $\mathrm{R}_{E}$ ) of the CPC perpendicular temperature falls with altitude which suggests that this region experiences low or no WPI [Nilsson et al. 2012, 2013]. This would lead us to introduce a third region where either the spectral density is low (which is unlikely) or the portion of wave activity that is efficient in heating the ions is low.

2. It is reasonable to assume that the change in spectral density with altitude is continuous and doesn't experience a sudden jump as would be the case if we changed the diffusion coefficients suddenly at a given altitude. Still, as a first study such an alteration may be assumed.

3. Spectral density data from DE-1 only cover altitudes up to $4.5 \mathrm{R}_{E}$ while spectral density data from Cluster only cover altitudes above $7 \mathrm{R}_{E}$, which leaves the mid altitude region with no spectral density data to deduce the correct diffusion coefficients. However, Waara et al. [2011]; Nilsson et al. [2012, 2013] only used Cluster orbits with apogeum towards the dayside polar cap which limited the data to altitudes above 7 Re. Cluster can however, provide data for lower altitudes which may give us insight into the mid altitude region.

It must also be noted that in each set of diffusion coefficients there are relatively high uncertainties which may correspond to different geophysical conditions.
This subject in addition to determining the effect of various combinations of diffusion coefficients and the effect of varying boundary conditions is the objective of an ongoing study.

In this study we have neglected the effect of centrifugal acceleration which is a very important acceleration mechanism especially at high altitudes. The effect of centrifugal acceleration on the outflow of ions above the polar cap is the subject of Abudayyeh et al. [2015, in press]. We have shown that centrifugal acceleration increases the parallel bulk velocity of the ions and decreases the parallel and perpendicular temperature.

In this study we have used a steady state 1-D model, which is usually referred to as the Barghouthi model. The applicability of the Barghouthi model has been discussed in several previous studies such as Barghouthi et al. [2012, 2014] and Nilsson et al. [2013]. The Barghouthi model has previously been shown to produce results that are similar to the observations [Barghouthi 2008, Barghouthi et al. 2011]. To make the results more reliable we have modified this model by: (a) subdividing the polar cap region into three trajectories to increase the spatial resolution; (b) replacing the radial field by the Tsyganenko T96 model which takes into consideration both internal and external sources of the magnetic field; and (c) replacing the previously used boundary conditions by more recent boundary conditions suggested by Nilsson et al. [2013]. In this model we have assumed that the ion outflow is along a field line without convection $(\mathrm{E} \times \mathrm{B}$ drift). Such a convection would lead to a drift from one region to another therefore mixing the plasmas of the two regions. By using this model we have therefore assumed low convection velocities which occur at quiet geomagnetic activity periods. Furthermore this model does not take into account the temporal variations of the outflow. Hence, this model is used to study the typical evolution of ion outflows for large 
distances along the geomagnetic field lines and the spatial and temporal transients are rather smoothed out. For a more accurate model that takes into consideration all these effects, we suggest that a 3-D model such as Barakat and Schunk [2006] needs to be used. However, due to their complexity, these models consume a great deal of time and resources, which makes conducting a parametric study like this impractical. Consequently, we suggest that a simplistic 1-D model such as the Barghouthi model be used to supply the more complicated 3-D models with the correct set of parameters for their implementation.

\section{References}

Abe, T., A.W. Yau, S. Watanabe, M. Yamada and E. Sagawa (2004). Long-term variation of the polar wind velocity and its implication for the ion acceleration process: Akebono/ suprathermal ion mass spectrometer observations, Journal of Geophysical Research: Space Physics, 109 (A9).

Abudayyeh, H.A., I.A. Barghouthi, R. Slapak and H. Nilsson (2015). Centrifugal acceleration at high altitudes above the polar cap: A Monte Carlo simulation, J. Geophys. Res. Space Physics, 120, 6409-6426; doi:10.1002/2015JA021325.

Arvelius, S., et al. (2005). Statistics of high-altitude and high-latitude $\mathrm{O}^{+}$ion outflows observed by Cluster/CIS, Annales Geophysicae, 23 (5), 1909-1916; doi:10.5194/angeo-23-1909-2005.

Barakat, A.R., and R.W. Schunk (2006). A three-dimensional model of the generalized polar wind, Journal of Geophysical Research: Space Physics, 111 (12), 114; doi:10.1029/2006JA011662.

Barghouthi, I.A. (1997). Effects of wave-particle interactions on $\mathrm{H}^{+}$and $\mathrm{O}^{+}$outflow at high latitude: A comparative study, Journal of Geophysical Research, 102 (A10), 22,065; doi:10.1029/96JA03293.

Barghouthi, I., A. Barakat and A. Persoon (1998). The Effects of Altitude-Dependent Wave Particle Interactions on the Polar Wind Plasma, Astrophysics and Space Science, 259 (2), 117-140; doi:10.1023 / A:1001 569207346.

Barghouthi, I.A., N.A. Qatanani and F.M. Allan (2003). Monte Carlo Simulation of Boltzmann Equation in Space Plasma at High Latitudes, Monte Carlo Methods and Applications, 9 (3); doi:10.1515/1569396033 22728978.

Barghouthi, I.A., and M.A. Atout (2006). Monte Carlo modeling of toroidal ion distributions and ion temperatures at high altitudes equatorward of the cusp: Effect of finite gyroradius, Journal of Geophysical Research, 111 (A3), A03202; doi:10.1029/2005JA011 437.
Barghouthi, I.A. (2008). A Monte Carlo study for ion outflows at high altitude and high latitude: Barghouthi model, Journal of Geophysical Research, 113 (A8), A08209; doi:10.1029/2008JA013274.

Barghouthi, I.A., S.H. Ghithan and H. Nilsson (2011). A comparison study between observations and simulation results of Barghouthi model for $\mathrm{O}^{+}$and $\mathrm{H}^{+}$ outflows in the polar wind, Annales Geophysicae, 29 (11), 2061-2079; doi:10.5194/angeo-29-2061-2011.

Barghouthi, I.A., H. Nilsson and S. Ghithan (2012). Comparison between the simulation results of Barghouthi model for ion outflows in the polar wind and auroral regions, Journal of the Association of Arab Universities for Basic and Applied Sciences, 12 (1), 1-10; doi:10.1016/j.jaubas.2012.08.001.

Barghouthi, I.A., H. Nilsson and S.H. Ghithan (2014). $\mathrm{O}^{+}$and $\mathrm{H}^{+}$ion heat fluxes at high altitudes and high latitudes, Annales Geophysicae, 32 (8), 1043-1057; doi:10.5194/angeo-32-1043-2014.

Bouhram, M., B. Klecker, W. Miyake, H. Reme, J. Sauvaud, M. Malingre, L. Kistler and A. Blagau (2004). On the altitude dependence of transversely heated $\mathrm{O}^{+}$distributions in the cusp/ cleft, Annales Geophysicae, 1787-1798; doi:10.5194/angeo-22-1787-2004.

Chang, T., and B. Coppi (1981). Lower hybrid acceleration and ion evolution in the suprauroral region, Geophysical Research Letters, 8 (12), 1253-1256; doi:10.1029/GL008i012p01253.

Chang, T., G.B. Crew, N. Hershkowitz, J.R. Jasperse, J.M. Retterer and J.D. Winningham (1986). Transverse acceleration of oxygen ions by electromagnetic ion cyclotron resonance with broad band left-hand polarized waves, Geophysical Research Letters, 13 (7), 636-639; doi:10.1029/GL013i007p00 636.

Cladis, J.B. (1986). Parallel acceleration and transport of ions from polar ionosphere to plasma sheet, Geophysical Research Letters, 13 (9), 893-896; doi:10.10 29/GL013i009p00893.

Crew, G.B., T. Chang, J.M. Retterer, W.K. Peterson, D.A. Gurnett and R.L. Huff (1990). Ion cyclotron resonance heated conics: Theory and observations, Journal of Geophysical Research, 95 (A4), 3959; doi:10.1029/JA095iA04p03959.

Demars, H.G., A.R. Barakat and R.W. Schunk (1996). Effect of centrifugal acceleration on the polar wind, Journal of Geophysical Research, 101 (A11), 24,565; doi:10.1029/96JA02234.

Gurnett, D.A., R.L. Huff, J.D. Menietti, J.L. Burch, J.D. Winningham and S.D. Shawhan (1984). Correlated low-frequency electric and magnetic noise along the auroral field lines, Journal of Geophysical Research, 89 (A10), 8971; doi:10.1029/JA089iA10p08971. 
Gurnett, D.A., and U.S. Inan (1988). Plasma wave observations with the Dynamics Explorer 1 spacecraft, Reviews of Geophysics, 26 (2), 285; doi:10.1029/RG $026 i 002$ p00285.

Horwitz, J.L., C.W. Ho, H.D. Scarbro, G.R. Wilson and T.E. Moore (1994). Centrifugal acceleration of the polar wind, Journal of Geophysical Research, 99 (A8), 15,051; doi:10.1029/94JA00924.

Huddleston, M.M., C.J. Pollock, M.P. Wuest, J.S. Pickett, T.E. Moore and W.K. Peterson (2000). Toroidal ion distributions observed at high altitudes equatorward of the cusp, Geophysical Research Letters, 27 (4), 469-472; doi:10.1029/1999GL010712.

Lundin, R., M. Yamauchi, J. Woch and G. Marklund (1995). Boundary layer polarization and voltage in the 14 MLT region, Journal of Geophysical Research, 100 (A5), 7587; doi:10.1029/94JA02523.

Maggiolo, R., et al. (2006). A multi-satellite study of accelerated ionospheric ion beams above the polar cap, Annales Geophysicae, 24 (6), 1665-1684.

Nilsson, H., et al. (2004). The structure of high altitude $\mathrm{O}^{+}$energization and outflow: a case study, Annales Geophysicae, 22 (7), 2497-2506; doi:10.5194/angeo22-2497-2004.

Nilsson, H., et al. (2006). Characteristics of high altitude oxygen ion energization and outflow as observed by Cluster: a statistical study, Annales Geophysicae, 24 (3), 1099-1112; doi:10.5194/angeo-241099-2006.

Nilsson, H., et al. (2008). An assessment of the role of the centrifugal acceleration mechanism in high altitude polar cap oxygen ion outflow, Annales Geophysicae, 26 (1), 145-157; doi:10.5194/ angeo-26-1452008.

Nilsson, H., E. Engwall, A. Eriksson, P.A. Puhl-Quinn and S. Arvelius (2010). Centrifugal acceleration in the magnetotail lobes, Annales Geophysicae, 28 (2), 569-576; doi:10.5194/ angeo-28-569-2010.

Nilsson, H., I.A. Barghouthi, R. Slapak, A.I. Eriksson and M. André (2012). Hot and cold ion outflow: Spatial distribution of ion heating, Journal of Geophysical Research, 117 (A11), A11201; doi:10.1029/ 2012JA017974.

Nilsson, H., I. A. Barghouthi, R. Slapak, A.I. Eriksson and M. André (2013). Hot and cold ion outflow: Observations and implications for numerical models, Journal of Geophysical Research: Space Physics, 118 (1), 105-117; doi:10.1029/2012JA017975.

Retterer, J., T. Chang, G. Crew, J. Jasperse and J. Winningham (1987). Monte Carlo modeling of ionospheric oxygen acceleration by cyclotron resonance with broad-band electromagnetic turbulence, Physical Review Letters, 59 (1), 148-151; doi:10.1103/
PhysRevLett.59.148.

Retterer, J.M., T. Chang and J.R. Jasperse (1994). Transversely accelerated ions in the topside ionosphere, Journal of Geophysical Research, 99 (A7), 13,189; doi:10.1029/93JA03570.

Schunk, R.W. (1977). Mathematical structure of transport equations for multispecies flows, Reviews of Geophysics, 15 (4), 429; doi:10.1029/RG015i004p00 429.

Slapak, R., H. Nilsson, M. Waara, M. André, G. Stenberg and I.A. Barghouthi (2011). $\mathrm{O}^{+}$heating associated with strong wave activity in the high altitude cusp and mantle, Annales Geophysicae, 29 (5), 931944; doi:10.5194/ angeo-29-931-2011.

Tsyganenko, N.A. (1995). Modeling the Earth's magnetospheric magnetic field confined within a realistic magnetopause, Journal of Geophysical Research, 100 (A4), 5599; doi:10.1029/94JA03193.

Tsyganenko, N.A. (1996). Effects of the solar wind conditions in the global magnetospheric configurations as deduced from data-based field models, In: E.J. Rolfe and B. Kaldeich (eds.), Proceedings of the 3rd International Conference on Substorms (Versailles, May 12-17, 1996), ESA SP-389, Paris: European Space Agency, p.181.

Waara, M., H. Nilsson, G. Stenberg, M. André, H. Gunell and H. Rème (2010). Oxygen ion energization observed at high altitudes, Annales Geophysicae, 28 (4), 907-916; doi:10.5194/ angeo-28-907-2010.

Waara, M., R. Slapak, H. Nilsson, G. Stenberg, M. André and I.A. Barghouthi (2011). Statistical evidence for $\mathrm{O}^{+}$energization and outflow caused by wave-particle interaction in the high altitude cusp and mantle, Annales Geophysicae, 29 (5), 945-954; doi:10.5194/ angeo-29-945-2011.

Waara, M., H. Nilsson, R. Slapak, M. André and G. Stenberg (2012). Oxygen ion energization by waves in the high altitude cusp and mantle, Annales Geophysicae, 30 (9), 1309-1314; doi:10.5194/ angeo-301309-2012.

Corresponding author: Hamza A. Abudayyeh, Al-Quds University, Department of Physics, Jerusalem, Palestine; email: abudayyehhamza@gmail.com.

(C) 2015 by the Istituto Nazionale di Geofisica e Vulcanologia. All rights reserved. 